\title{
Botulinum neurotoxin for the treatment of blepharospasm, cervical dystonia, adult spasticity, and headache
}

Payment policy perspectives

\section{Raghav Govindarajan, MD; Katie M. Shepard; Constantine Moschonas, MD; Jack J. Chen, PharmD, BCPS, CGP}

\begin{abstract}
This article is presented as a companion to the American Academy of Neurology guideline update on the use of botulinum neurotoxin (BoNT) for the treatment of blepharospasm, cervical dystonia, adult spasticity, and headache. Whereas the guideline update provides clarity on the efficacy of different BoNT-branded preparations for the 4 listed indications, this companion piece identifies ambiguities in the evidence for efficacy among various brand names for a given clinical indication, their dosing equivalencies, as well as different clinical indications. This article provides guidance and background information to reduce obstacles for third party payment, especially when uncertainties exist and levels of evidence are lower. Neurol Clin
\end{abstract}

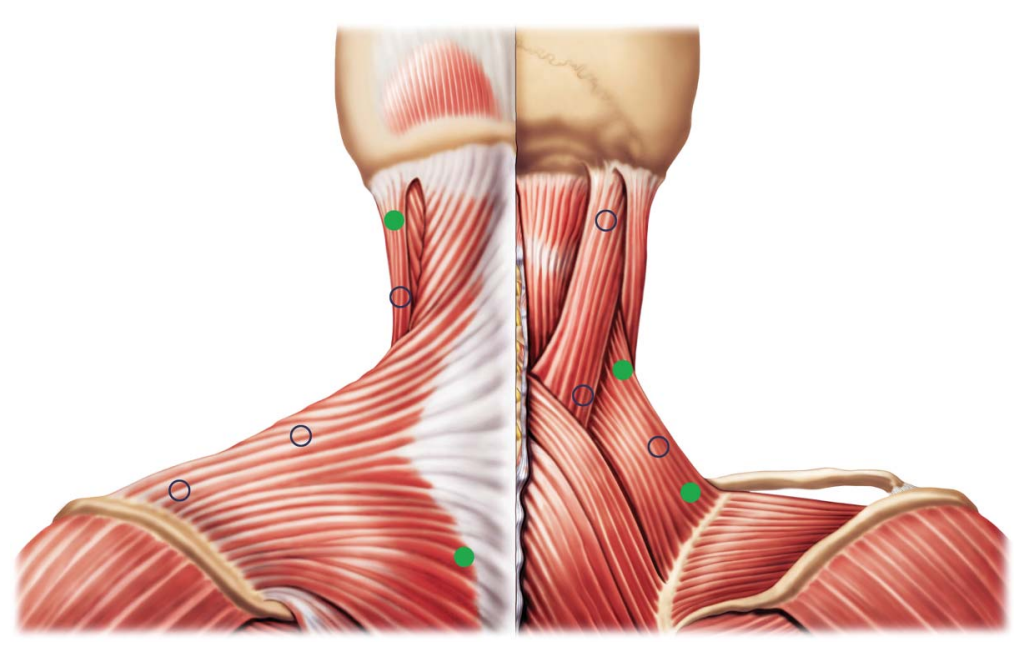

Pract 2016;6:281-286

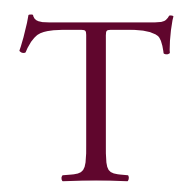

he first American Academy of Neurology (AAN) guideline on the use of botulinum toxin (BoNT), published in 2008, focused predominantly on the pharmacology and immunology of the toxin. ${ }^{1-3}$ The guideline also reviewed a variety of clinical indications, with recommendations of efficacy based on the toxin as a whole. Furthermore, there was insufficient evidence to recommend the use of BoNT for chronic migraine.

Since then, there has been more evidence on the efficacy of different brand formulations for different indications as well greater understanding of the differences in pharmacology and immunology between the toxin A and B serotypes and branded formulations. However, there are

University of Missouri (RG), Columbia, MO; American Academy of Neurology (KMS), Minneapolis, MN; Four Peaks Neurology (CM), Scottsdale, AZ; and Marshall B. Ketchum University (JJC), Fullerton, CA.

Funding information and disclosures are provided at the end of the article. Full disclosure form information provided by the authors is available with the full text of this article at Neurology.org/cp.

Approved by the Payment Policy Subcommittee on December 14, 2014; by the Medical Economics and Management Committee on March 1, 2016; and by the AAN Board of Directors on April 7, 2016.

Correspondence to: kshepard@aan.com 


\section{There are still multiple gaps in evidence for efficacy among various brand names for a given clinical indication, their dosing equivalencies, as well as different clinical indications.}

no consistent studies that establish dosing equivalencies among these brands. Further evidence has emerged on the efficacy of BoNT for chronic migraine. ${ }^{4,5}$

The newly revised AAN guideline ${ }^{6}$ focuses on the use of BoNT in 4 clinical indications, including chronic migraine. Furthermore, for each of these indications, the guideline provides evidence of efficacy for each brand currently approved in the United States rather than the toxin class as a whole. Although traditionally payment policies covered BoNT A and B either separately or under a single policy, future policies might be more inclined to cover a specific brand for a given clinical indication. It is therefore prudent that providers be aware of BoNT brands, respective indications, and current payer policies.

Although the update to the original BoNT guideline does provide brand-specific evidence for the 4 described clinical indications, there are still multiple gaps in evidence for efficacy among various brand names for a given clinical indication, their dosing equivalencies, as well as different clinical indications. Although this could spur future research, payers still expect data and justification to satisfy current covered diagnoses (i.e., on-label and off-label indications). In this article, we discuss solutions to coverage situations that can result from incomplete evidence.

\section{Blepharospasm}

Two Class I comparative effectiveness trials of onabotulinum toxin A with incobotulinum toxin A have provided evidence of equal efficacy between the 2 toxins. ${ }^{7}$ Similarly, 1 Class II comparative effectiveness trial between abobotulinumtoxin $\mathrm{A}$ and onabotulinum toxin $\mathrm{A}$ has provided evidence of equal efficacy. ${ }^{7}$ There are no data on the efficacy of rimabotulinum toxin $\mathrm{B}$, so providers who choose to use it for this indication may need to be prepared to defend their BoNT choice in the event of a payer denial. If higher doses than those specified in the product insert are administered, the responsibility falls on providers to justify and clearly document the need in certain patient populations.

\section{Chronic migraine}

Two Class I studies comparing onabotulinumtoxin A with placebo have provided evidence of efficacy by reducing the number of headache days in patients with chronic migraine. ${ }^{4,5}$ There have been no comparative effectiveness trials between BoNT and oral prophylactic drugs used to treat chronic migraine. Given the high costs of BoNT and the high prevalence of chronic migraine, some payers (e.g., Anthem, BCBS, Highmark) have instituted a step therapy in their treatment (e.g., oral prophylactic would have to be tried and fail before onabotulinumtoxinA could be offered as a treatment option). In such cases, it is imperative that providers clearly document compliance with step therapy criteria and obtain prior authorization before they start using onabotulinumtoxinA for treatment of chronic migraine. ${ }^{8}$ It is also prudent that a headache diary be maintained by the patient to show efficacy in case payers question its benefit and ultimately deny coverage. Data for other BoNT formulations in the treatment of chronic migraine did not meet inclusion criteria for the updated guidelines; before using other BoNT brands, providers should check with payers for migraine coverage.

For episodic migraine, the guideline clearly states that use of onabotulinumtoxinA is ineffective. Providers should not be surprised if payers do not cover BoNT for treatment of episodic 


\section{Payers often adopt BoNT package insert statements on maximum allowable doses and injection frequency for determination of} coverage.

migraine. It is important to clearly document that the patient's headache symptoms meet the International Classification of Headache Disorders definition of chronic migraine (e.g., $\geq 15$ days per month) to prevent denials. ${ }^{9}$

\section{Cervical dystonia}

Based on the guideline findings, all brands of BoNT should enjoy payer coverage for the treatment of cervical dystonia. Although comparative dosing efficacies and equivalencies among different brands are not clearly established, we do not foresee any payer denials when there is a medical indication to change from 1 brand to another given the extensive evidence of efficacy of BoNTs for this indication. Additional research in this area using meta-analytical methods, such as mixed-treatment comparisons, will introduce additional data for evidence-based statements on the comparative efficacy of the BoNT brands.

\section{Adult spasticity}

Abobotulinumtoxin A and onabotulinumtoxin A have been shown to reduce upper-limb hypertonia with improvement of passive function. ${ }^{10,11}$ OnabotulinumtoxinA was found through comparative effectiveness studies to be superior to tizanidine in improving wrist flexor tone. ${ }^{12}$ Some payers may have a step therapy requirement or require documentation of failure of other standard medical therapies. Providers should periodically keep up with local payer policies and reimbursement changes. ${ }^{13}$ For reduction of lower-limb hypertonia, abobotulinumtoxinA and onabotulinumtoxinA can be offered as treatment options. ${ }^{14,15}$

\section{Dosing}

Dosing for BoNT drugs, as listed on the product insert, may not be sufficient for patients with cervical dystonia for their initial — and especially subsequent—injections. Dosing should be based on provider experience and the patient's functional goals for initial and subsequent treatments. Payers often adopt BoNT package insert statements on maximum allowable doses and injection frequency for determination of coverage. To reduce likelihood of denials, providers should be familiar with branded product labeling parameters for dosage and intervals. Documenting responsiveness or lack of responsiveness to make dosing changes including escalation is good clinical practice and will demonstrate medical necessity, especially if doses or injection intervals fall outside the payer's allowable parameters. Payers may appreciate a written procedure note that indicates the injected sites and notes the specific dosages injected at each site. This is also good clinical practice, as it will better enable reproduction of injections at future visits.

\section{What if my payer doesn't have a coverage policy on botulinum toxins?}

Correct coding (using Current Procedural Terminology [CPT] and ICD-10-CM) is essential for successful integration of BoNT therapy into clinical practice, and it is important for providers to periodically keep up with policies and reimbursement changes. ${ }^{13}$ Each of the BoNT manufacturers has specialists to assist in reimbursement issues (e.g., prior authorization, billing and coding, insurance verification, local coverage policy support, claims denials and appeals). Medicare, Medicaid, and private insurances cover BoNT treatment for on- and offlabel uses that are considered medically necessary. Based on a review of current payer policies and anecdotal experience, if BoNT is used for an off-label condition in which it is considered 


\section{Table CPT codes for botulinum toxin injections}

$64615 \quad$ Chemodenervation of muscle(s); muscle(s) innervated by facial, trigeminal, cervical spinal and accessory nerves, bilateral (e.g., for chronic migraine)

64616 Chemodenervation of muscle(s); neck muscle(s), excluding muscles of the larynx, unilateral (e.g., for cervical dystonia, spasmodic torticollis)

64617 Chemodenervation of muscle(s); larynx, unilateral, percutaneous (e.g., for spasmodic dysphonia), includes guidance by needle electromyography, when performed

64642 Chemodenervation of one extremity; 1-4 muscle(s)

$\mathbf{6 4 6 4 3}^{\mathrm{a}} \quad$ Chemodenervation of one extremity; each additional extremity, 1-4 muscle(s), (Use 64613 in conjunction with 64642, 64644)

64644 Chemodenervation of one extremity; 5 or more muscle(s)

64645 $\quad$ Chemodenervation of one extremity; each additional extremity, 5 or more muscle(s), (Use 64645 in conjunction with 64644)

$64646 \quad$ Chemodenervation of trunk muscle(s); 1-5 muscle(s)

64647 Chemodenervation of trunk muscle(s); 6 or more muscle(s)

$76942 \quad$ Ultrasonic guidance for needle placement (e.g., biopsy, aspiration, injection, localization device), imaging supervision and interpretation

$\mathbf{9 5 8 7 3}^{\mathbf{a}} \quad$ Electrical stimulation for guidance in conjunction with chemodenervation (List separately in addition to code for primary procedure)

95874 ${ }^{\text {a }} \quad$ Needle electromyography for guidance in conjunction with chemodenervation (List separately in addition to code for primary procedure)

AMA CPT Copyright Statement: CPT codes, descriptions, and other data are copyright 2015 American Medical Association. All rights reserved. Applicable FARS/DFARS clauses apply.

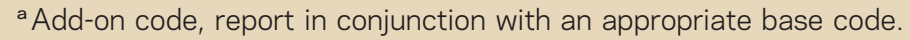

standard of care (i.e., a covered diagnosis), it will generally be approved. Although private insurance policies are generally universal, Medicare policies can vary from state to state. Providers should keep up-to-date with their state Medicare policies.

Novitas Solutions-the Part B Medicare administrative contractor for several statesannounced in July 2013 that it had retired its coverage policy on BoNTs retroactively effective to January $18,2013 .{ }^{16}$ It is also possible that some payers might not have updated their policies and in rare cases not even have a policy in place on the use of BoNT. It is imperative that providers check their patients' payer policies periodically.

With traditional Medicare, no prior authorization is needed for BoNT, and payment will occur if the procedure is covered on the state's policy and if proper linkage between ICD10-CM code and CPT code (table) is documented. For patients with private insurance or Health Maintenance Organization Medicare/Medicaid, prior authorization for BoNT should be obtained, even for on-label indications. A letter of medical necessity should be included with the prior authorization request. Clear and consistent documentation of medical necessity and therapeutic benefit is necessary, especially in cases in which BoNT is needed long-term or should a payer audit occur.

\section{Other considerations}

Improvements in muscle hypertonicity and functional improvement For some indications in the guideline, the studies included are characterized by dissimilar endpoint assessments for muscle hypertonicity and functional outcomes, which can make direct comparisons of BoNT brands challenging. Future studies and methodologies (e.g., mixed-treatment comparison) will have to be designed to address this important question. From a practice perspective, documentation of medical necessity for BoNT treatment in the 4 indications is essential. For instance, for initiation and long-term treatment of spasticity and muscle hypertonicity, providers should 
document BoNT use for reduction in hypertonicity and/or functional gains (e.g., pain relief, to assist in posturing and walking, to prevent joint contracture or future growth, to allow better range of motion, to permit better physical therapy or use of other therapeutic modalities, and to reduce severe spasm in order to provide adequate perineal hygiene). Evidence of such documentation will also avoid issues should a payer audit occur.

Using EMG or ultrasound for guided injections EMG, muscle stimulation, and ultrasound can be used independently or together based on clinical necessity and are reimbursable. For example, Medicare allows for EMG or electrical stimulation to be performed with ultrasound guidance. Use of these techniques can optimize clinical efficacy. Although studies have shown that EMG- and ultrasonography-guided injection of BoNT is more likely to reach the targeted muscle as well as its endplate in treatment of spasticity, ${ }^{17,18}$ it remains unclear whether injections using EMG or ultrasonography guidance result in greater functional improvement than nonguided injections. Although generally payers will not question EMG or ultrasound for BoNT, there are no clear payer policies on the use of guided injections, and their use depends on the experience and comfort level of the individual providers.

\section{CONCLUSION}

Many providers are adding or already offer BoNT procedures to their practice. With the updated guidelines, providers are now current with the latest evidence base for use of each BoNT formulation for various neurologic indications. Once a clinically appropriate patient has been identified, knowledge of reimbursement elements is essential for successful integration of BoNT procedures into clinical practice. The BoNT therapeutic space is evolving at a rapid pace and providers are encouraged to stay up-to-date with changes in the scientific literature and reimbursement landscape.

\section{REFERENCES}

1. Assessment: botulinum neurotoxin for the treatment of spasticity (an evidence-based review): report of the Therapeutics and Technology Assessment Subcommittee of the American Academy of Neurology. Neurology 2008;70:1691-1698.

2. Assessment: botulinum neurotoxin for the treatment of movement disorders (an evidence-based review): report of the Therapeutics and Technology Assessment Subcommittee of the American Academy of Neurology. Neurology 2008;70:1699-1706.

3. Assessment: botulinum toxin in the treatment of autonomic disorders and pain (an evidence-based review): report of the Therapeutics and Technology Assessment Subcommittee of the American Academy of Neurology. Neurology 2008;70:1707-1714.

4. Aurora SK, Dodick DW, Turkel CC, et al. OnabotulinumtoxinA for treatment of chronic migraine: results from the double-blind, randomized, placebo-controlled phase of the PREEMPT 1 trial. Cephalalgia 2010;30:793-803.

5. Diener HC, Dodick DW, Aurora SK, et al. OnabotulinumtoxinA for treatment of chronic migraine: results from the double-blind, randomized, placebo-controlled phase of the PREEMPT 2 trial. Cephalalgia 2010;30:804-814.

6. Simpson DM, Hallett M, Ashman EJ, et al. Practice guideline update summary: botulinum neurotoxin for the treatment of blepharospasm, cervical dystonia, adult spasticity, and headache: report of the Guideline Development Subcommittee of the American Academy of Neurology. Neurology Epub 2016 Apr 18.

7. Hallett M, Albanese A, Dressler D, et al. Evidence-based review and assessment of botulinum neurotoxin for the treatment of movement disorders. Toxicon 2013;67:94-114.

8. United HealthCare Botulinum Toxins A and B Drug Policy. Available at: https:/www. unitedhealthcareonline.com/ccmcontent/ProviderII/UHC/en-US/Assets/ProviderStaticFiles/ ProviderStaticFilesPdf/Tools\%20and\%20Resources/Policies\%20and\%20Protocols/Medical\%20Policies/ Drug\%20Policies/Botulinum_toxin_policy.pdf. Accessed June 30, 2015.

9. Headache Classification Committee of the International Headache Society. The International Classification of Headache Disorders, 3rd edition (beta version). Cephalalgia 2013;33:Section 1.3. Available at: http://www.ihs-classification.org/_downloads/mixed/International-Headache-Classification-III-ICHD-III2013-Beta.pdf. Accessed June 30, 2015. 
10. McCrory P, Turner-Stokes L, Baguley IJ, et al. Botulinum toxin A for treatment of upper limb spasticity following stroke: a multi-centre randomized placebo-controlled study of the effects on quality of life and other person-centred outcomes. J Rehabil Med 2009;41:536-544.

11. Shaw LC, Price CI, van Wijck FM, et al; BoTULS Investigators. Botulinum toxin for the upper limb after stroke (BoTULS) trial: effect on impairment, activity limitation, and pain. Stroke 2011;42: 1371-1379.

12. Simpson DM, Gracies JM, Yablon SA, Barbano R, Brashear A; BoNT/TZD Study Team. Botulinum neurotoxin versus tizanidine in upper limb spasticity: a placebo-controlled study. J Neurol Neurosurg Psychiatry 2009;80:380-385.

13. Taylor M. Primer on botulinum toxin billing and coding: what neurologists need to know for 2014 . Practical Neurology 2014. Available at: http://practicalneurology.com/2014/03/primer-on-botulinumtoxin-billing-and-coding-what-neurologists-need-to-know-for-2014/. Accessed June 30, 2015.

14. Gusev YI, Banach M, Simonow A, et al. Efficacy and safety of botulinum type A toxin in adductor spasticity due to multiple sclerosis. J Musculoskel Pain 2008;16:175-188.

15. Kaji R, Osako Y, Suyama K, Maeda T, Uechi Y, Iwasaki M; GSK1358820 Spasticity Study Group. Botulinum toxin type A in post-stroke lower limb spasticity: a multicenter, double-blind, placebocontrolled trial. J Neurol 2010;257:1330-1337.

16. Novitas Solutions. Announcement. Available at: http://www.novitas-solutions.com/cs/idcplg? IdcService $=$ GET_DYNAMIC_CONVERSION $\&$ RevisionSelectionMethod $=$ LatestReleased $\&$ $\mathrm{dDocName}=00006151 \&$ proto $=$ http\&domain $=$ www. novitas-solutions. com \&space $=$ MedicareJH. Accessed June 30, 2015.

17. Picelli A, Lobba D, Midiri A, et al. Botulinum toxin injection into the forearm muscles for wrist and fingers spastic overactivity in adults with chronic stroke: a randomized controlled trial comparing three injection techniques. Clin Rehabil 2014;28:232-242.

18. Lee LH, Chang WN, Chang CS. The finding and evaluation of EMG-guided BOTOX injection in cervical dystonia. Acta Neurol Taiwan 2004;13:71-76.

Received June 16, 2015. Accepted in final form September 10, 2015.

\section{AUTHOR CONTRIBUTIONS}

Raghav Govindarajan: study concept and design, analysis and interpretation, critical revision of the manuscript for important intellectual content. Katie Shepard: study concept and design, critical revision of the manuscript for important intellectual content. Constantine Moschonas: critical revision of the manuscript for important intellectual content. Jack J. Chen: study analysis and interpretation, critical revision of the manuscript for important intellectual content.

\section{ACKNOWLEDGMENT}

The authors thank members of the American Academy of Neurology Payment Policy Subcommittee as well as the authors of the AAN's guideline document for their support of the concept and review of early versions of the manuscript.

\section{STUDY FUNDING}

No targeted funding reported.

\section{DISCLOSURES}

R. Govindarajan reports no disclosures. K. M. Shepard is a full-time employee of the American Academy of Neurology. C. Moschonas reports no disclosures. J. J. Chen serves on a scientific advisory board for Ipsen and serves on the editorial board of the Journal of Toxins. Full disclosure form information provided by the author is available with the full text of this article at Neurology.org/cp.

\section{Related articles from AAN physician and patient resources}

\section{Continuum ${ }^{\circledR}$ - ContinuumJournal.com}

Coding for the Use of Botulinum Toxin for Movement Disorders

October 2013;19:1406-1415. 


\title{
Neurology ${ }^{\circ}$ Clinical Practice
}

\author{
Botulinum neurotoxin for the treatment of blepharospasm, cervical dystonia, adult \\ spasticity, and headache: Payment policy perspectives \\ Raghav Govindarajan, Katie M. Shepard, Constantine Moschonas, et al. \\ Neurol Clin Pract 2016;6;281-286 Published Online before print April 18, 2016 \\ DOI 10.1212/CPJ.0000000000000243
}

This information is current as of April 18, 2016

Updated Information \&
Services
References
Citations
Subspecialty Collections

Subsecialty Collections

Permissions \& Licensing

Reprints including high resolution figures, can be found at:

http://cp.neurology.org/content/6/3/281.full.html

This article cites 13 articles, 2 of which you can access for free at: http://cp.neurology.org/content/6/3/281.full.html\#\#ref-list-1

This article has been cited by 2 HighWire-hosted articles: http://cp.neurology.org/content/6/3/281.full.html\#\#otherarticles

This article, along with others on similar topics, appears in the following collection(s):

All Headache

http://cp.neurology.org//cgi/collection/all_headache

All Practice Management

http://cp.neurology.org//cgi/collection/all_practice_management Billing

http://cp.neurology.org//cgi/collection/billing

\section{Botulinum toxin}

http://cp.neurology.org//cgi/collection/botulinum_toxin

Insurance

http://cp.neurology.org//cgi/collection/insurance

Information about reproducing this article in parts (figures,tables) or in its entirety can be found online at:

http://cp.neurology.org/misc/about.xhtml\#permissions

Information about ordering reprints can be found online:

http://cp.neurology.org/misc/addir.xhtml\#reprintsus

Neurol Clin Pract is an official journal of the American Academy of Neurology. Published continuously since 2011, it is now a bimonthly with 6 issues per year. Copyright $\odot 2016$ American Academy of Neurology. All rights reserved. Print ISSN: 2163-0402. Online ISSN: 2163-0933.

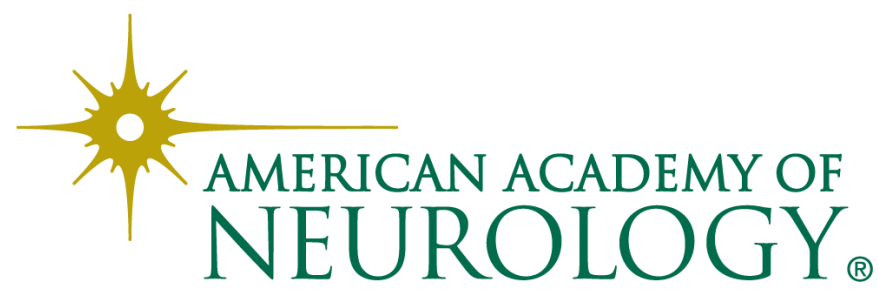

